\title{
Yersinia pseudotuberculosis and Yersinia pestis show increased outer membrane permeability to hydrophobic agents which correlates with lipopolysaccharide acyl-chain fluidity
}

\author{
José-Antonio Bengoechea, ${ }^{1,2}$ Klaus Brandenburg, ${ }^{2}$ Ulrich Seydel, ${ }^{2}$ \\ Ramón Díaz ${ }^{1}$ and Ignacio Moriyón ${ }^{1}$
}

Author for correspondence: Ignacio Moriyón. Tel: +34 48 425600. Fax: +34 48425649.
e-mail: imoriyon@unav.es

1 Departamento de Microbiología, Universidad de Navarra, Aptdo. 177, 31080 Pamplona, Spain

2 Forschungzentrum Borstel, Division of Biophysics, Borstel, Germany
The hydrophobic probe $\boldsymbol{N}$-phenyl-1-naphthylamine accumulated less in nonpathogenic Yersinia spp. and non-pathogenic and pathogenic Yersinia enterocolitica than in Yersinia pseudotuberculosis or Yersinia pestis. This was largely due to differences in the activity of efflux systems, but also to differences in outer membrane permeability because uptake of the probe in KCN/arsenate-poisoned cells was slower in the former group than in $Y$. pseudotuberculosis and $Y$. pestis. The probe accumulation rate was higher in $Y$. pseudotuberculosis and $Y$. pestis grown at $37^{\circ} \mathrm{C}$ than at $26^{\circ} \mathrm{C}$ and was always highest in $Y$. pestis. These yersiniae had LPSs with shorter polysaccharides than $Y$. enterocolitica, particularly when grown at $37^{\circ} \mathrm{C}$. Gel $\leftrightarrow$ liquid-crystalline phase transitions $\left(T_{c} 28-31^{\circ} \mathrm{C}\right)$ were observed in LPS aggregates of $Y$. enterocolitica grown at 26 and $37^{\circ} \mathrm{C}$, with no differences between nonpathogenic and pathogenic strains. $Y$. pseudotuberculosis and $Y$. pestis LPSs showed no phase transitions and, although the fluidity of LPSs of $Y$. pseudotuberculosis and $Y$. enterocolitica grown at $26^{\circ} \mathrm{C}$ were close below the $T_{c}$ of the latter, they were always in a more fluid state than $Y$. enterocolitica LPS. Comparison with previous studies of Salmonella choleraesuis subsp. choleraesuis serotype minnesota rough LPS showed that the increased fluidity and absence of transition of $Y$. pseudotuberculosis and $Y$. pestis LPSs cannot be explained by their shorter polysaccharides and suggested differences at the lipid A/core level. It is proposed that differences in LPS-LPS interactions and efflux activity explain the above observations and reflect the adaptation of Yersinia spp. to different habitats.

Keywords: Yersinia, outer membrane, permeability, lipopolysaccharide

\section{INTRODUCTION}

The yersiniae are members of the Enterobacteriaceae and include both pathogenic and non-pathogenic species and biotypes. Whereas Yersinia intermedia, Yersinia mollaretii, Yersinia rohdei, Yersinia bercovieri, Yersinia frederiksenii and Yersinia kristensenii are merely opportunistic pathogens found mostly in the environment, Yersinia enterocolitica includes both non-pathogenic

Abbreviations: DOC, sodium deoxycholate; FTIR, Fourier-transformed infrared spectroscopy; Kdo, 3-deoxy-D-manno-2-octulosonic acid; NPN, $\mathrm{N}$ phenyl-1-naphthylamine; OM, outer membrane. and pathogenic biogroups and Yersinia pseudotuberculosis and the closely related Yersinia pestis are characteristically pathogenic (Bercovier \& Mollaret, 1984). Y. enterocolitica and Y. pseudotuberculosis cause intestinal infections in humans but they differ in the degree of invasiveness which, although limited in both, is greater in the latter (Brubaker, 1991). In contrast, $Y$. pestis enters the body by routes other than the gut, it is very invasive and characteristically multiplies within non-activated macrophages (Brubaker, 1991; Straley \& Harmon, 1984a, b). Pathogenic yersiniae carry a virulence plasmid (pYV) and many virulence-related properties are regulated by environmental factors such as temperature, $\mathrm{pH}, \mathrm{Ca}^{2+}$ availability and contact with 
eukaryotic cells, at both plasmid and chromosome levels (Brubaker, 1991; Cornelis \& Wolf-Watz, 1997; Straley $\&$ Perry, 1995).

Because of the importance of outer membranes (OMs) in the interaction of Gram-negative bacteria with the environment, we have hypothesized that the wide differences in habitat and pathogenicity between yersiniae should be reflected in their OM properties (Bengoechea et al., 1996). In a previous study, we found that plasmid-bearing $\left(\mathrm{pYV}^{+}\right)$and plasmid-cured $\left(\mathrm{pYV}^{-}\right)$ pathogenic $Y$. enterocolitica had OMs resistant to polycations compared to Y. enterocolitica biogroup 1A (environmental and non-pathogenic) and enteropathogenic Escherichia coli. In addition, when grown with $\mathrm{Mg}^{2+}$ but with no $\mathrm{Ca}^{2+}$ available, pathogenic $Y$. enterocolitica biogroups displayed a marked increase in OM permeability to hydrophobic probes which was not observed in the non-pathogenic biogroup or in E. coli (Bengoechea et al., 1996). We have extended these studies to Y. pseudotuberculosis and Y. pestis, the more invasive of the pathogenic yersiniae, and considered the influence of both the OM barrier and possible efflux systems. We report here that there are large differences between Yersinia spp. in their ability to repel hydrophobic compounds and that such differences correlate with the degree of acyl-chain fluidity in their respective LPS. We also present indirect evidence suggesting differences in efflux systems between yersiniae.

\section{METHODS}

Bacterial strains and growth conditions. The strains used (Table 1) were stored in skimmed milk at $-80^{\circ} \mathrm{C}$ and inocula were taken directly from these frozen seeds to minimize loss of pYV for all experiments (Bengoechea et al., 1996). Selection of the $\mathrm{pYV}^{+}$(virulent) and plasmid-cured $\mathrm{pYV}^{-}$(avirulent) isogenic strains was performed on Congo Red/magnesium/ oxalate medium (Riley \& Toma, 1989). For permeability studies, cells were grown in standard tryptic soy broth in sidearm flasks on an orbital shaker (250 r.p.m.) at 26 or $37^{\circ} \mathrm{C}$ and growth was monitored by measuring $\mathrm{OD}_{540}$. To test the effect of pYV expression, the cells were grown in magnesium/ oxalate medium (Riley \& Toma, 1989). Exponential phase cells were harvested $\left(5000 \mathrm{~g}, 20 \mathrm{~min}, 5^{\circ} \mathrm{C}\right)$ and resuspended immediately in the appropriate buffer or broth (see below). For LPS extraction, Y. pseudotuberculosis and Y. enterocolitica were grown in tryptic soy broth and Y. pestis in standard brain-heart infusion in 21 flasks ( $800 \mathrm{ml}$ per flask) on an orbital shaker (200 r.p.m.) for $24 \mathrm{~h}$ at 26 or $37^{\circ} \mathrm{C}$.

Sensitivity to hydrophobic antibiotics and dyes. Serial dilutions of the appropriate agents (Table 2) were made in sterile 96-well polystyrene plates (Nunc) with Mueller-Hinton broth as the diluent. A fresh suspension (approx. $1.25 \times 10^{6}$ c.f.u. $\mathrm{ml}^{-1}$ ) of exponential phase cells grown at $37^{\circ} \mathrm{C}$ was prepared in the same broth and $100 \mu$ laliquots were pipetted into each well. Plates were incubated at $37^{\circ} \mathrm{C}$ for $16 \mathrm{~h}$ and the lowest concentration of each agent that completely inhibited visible growth was taken as its MIC.

$\boldsymbol{N}$-Phenyl-1-naphthylamine (NPN) uptake. NPN is a hydrophobic fluorescent dye which, unless hindered, partitions into the hydrophobic moiety of membranes with a simultaneous increase in quantum yield and which has been proven useful in
OM permeability studies (Bengoechea et al., 1996; Loh et al., 1984; Freer et al., 1996; Martínez de Tejada \& Moriyón, 1993; Träuble \& Overath, 1973). Exponentially growing cells were resuspended in $2 \mathrm{mM}$ HEPES ( $\mathrm{pH} 7 \cdot 2$ ) with or without $\mathrm{KCN}$ plus sodium arsenate $(1-25 \mathrm{mM}$ for both inhibitors; see Results) to an $\mathrm{OD}_{600}$ of $0 \cdot 5$. Cells were then transferred to $1 \mathrm{~cm}$ diameter fluorimetric cuvettes, NPN $(500 \mu \mathrm{M}$ in acetone) was added to a final concentration of $10 \mu \mathrm{M}$ and changes in fluorescence were monitored with an LS-50 fluorimeter (Perkin-Elmer; excitation $350 \mathrm{~nm}$, emission $420 \mathrm{~nm}$, slit width $2.5 \mathrm{~nm})$. Measurements were performed at the growth temperature and results were expressed as relative fluorescence units (RFU) (Bengoechea et al., 1996). Measurements were repeated at least twice with each of two independently grown batches of bacteria.

Sensitivity to deoxycholate. Late exponential phase cells were resuspended to an $\mathrm{OD}_{450}$ of 0.21 using saline with or without $1 \mathrm{mM}$ sodium arsenate $/ 1 \mathrm{mM} \mathrm{KCN}$ and $2 \mathrm{ml}$ was transferred to tubes containing increasing concentrations of sodium deoxycholate (DOC) (see Results). After $1 \mathrm{~h}$ at the growth temperature, lysis was measured as the percentage $\mathrm{OD}_{450}$ of bacterial suspensions incubated without DOC. All experiments were performed in duplicate with two independently grown batches of bacteria.

LPS preparations. The LPSs of Y. enterocolitica PR O:1,6, WA 289 O: 8 and WE 245/92 O:3, Y. pseudotuberculosis WE $23 / 90$ and Y. pestis KIM were obtained from the water phase of a water/phenol extract as described by Sprott et al. (1994) and fractionation of the LPSs into the water but not the phenol phase was confirmed. Crude LPSs were dispersed $\left(10 \mathrm{mg} \mathrm{ml}^{-1}\right)$ in $0.8 \% \mathrm{NaCl} / 0 \cdot 05 \% \mathrm{NaN}_{3} / 0 \cdot 1 \mathrm{M}$ Tris $/ \mathrm{HCl}(\mathrm{pH} 7)$, digested with nucleases and proteinase $\mathrm{K}$ as described previously (Bengoechea et al., 1996) and the purified LPSs were sedimented by ultracentrifugation $(100000 \mathrm{~g}, 6 \mathrm{~h})$ and freezedried. The 3-deoxy-D-manno-2-octulosonic acid (Kdo) content was determined by the thiobarbituric acid method modified to correct interference due to deoxysugars (DíazAparicio et al., 1993) and protein content was determined as described by Markwell et al. (1978) using serum albumin as standard. SDS-PAGE and silver staining for LPS were performed as described by Tsai \& Frasch (1982). As a reference, the deep R-LPS from Salmonella choleraesuis subsp. choleraesuis serotype minnesota (hereafter referred to as S. minnesota) Re595 (obtained by the phenol/chloroform/ petroleum ether method) was included in all gels. Absence of phospholipids was shown by analysis of chloroform/ methanol extracts of the LPS preparations by conventional methods (Thiele \& Schwinn, 1973).

Determination of the gel $\leftrightarrow$ liquid-crystalline $(\beta \leftrightarrow \alpha)$ phase transition of Yersinia LPSs. The $\beta \leftrightarrow \alpha$ phase transition of the acyl chains of LPS from a well-ordered (gel) into a fluid (liquid-crystalline) state at a lipid-specific temperature, $T_{c}$, was measured by two methods : Fourier-transformed infrared spectroscopy (FTIR) and fluorescence polarization spectroscopy. This allows the determination of the acyl-chain fluidity - inversely proportional to the state of order - which is a measure of the mobility of the hydrocarbon chains at a given temperature. Thus, in the gel phase, well below $T_{\mathrm{c}}$, the acyl chains are in a highly ordered, rigid state and in the liquidcrystalline phase, well above $T_{c}$, they are highly disordered. The natural salt forms of the LPSs were used in the experiments described below.

(i) FTIR. To ensure homogeneity, LPS dispersions were prepared in $2.5 \mathrm{mM}$ HEPES ( $\mathrm{pH} \mathrm{7.0)}$ ) at room temperature, incubated at $56^{\circ} \mathrm{C}$ for $15 \mathrm{~min}$, stirred and cooled to $4{ }^{\circ} \mathrm{C}$. This heatingcooling step was repeated three times and suspensions were 
Table 1. Bacterial strains used

\begin{tabular}{|c|c|c|}
\hline Strain & Serogroup (biogroup) & Characteristics \\
\hline Y. kristensenii WA 396 & $O: 11,24$ & Non-pathogenic \\
\hline Y. intermedia WA 9/91 & $0: 40$ & Non-pathogenic \\
\hline Y. frederiksenii WA $99 / 92$ & $0: 48$ & Non-pathogenic \\
\hline Y. bercovieri WS $68 / 91$ & $0: 73,58$ & Non-pathogenic \\
\hline Y. mollaretii WA $19 / 88$ & $0: 75$ & Non-pathogenic \\
\hline Y. rohdei WA $9 / 90$ & $0: 76$ & Non-pathogenic \\
\hline Y. enterocolitica PR & $O: 1,6(1 \mathrm{~A})$ & Environmental (non-pathogenic) \\
\hline Y. enterocolitica MA 279 & $0: 5(1 \mathrm{~A})$ & Environmental (non-pathogenic) \\
\hline Y. enterocolitica MA 280 & $0: 6(1 \mathrm{~A})$ & Environmental (non-pathogenic) \\
\hline Y. enterocolitica MA 282 & $\mathrm{O}: 7,8,13,19(1 \mathrm{~A})$ & Environmental (non-pathogenic) \\
\hline Y. enterocolitica IP 135 & $O: 1,2 \mathrm{a}, 3(3)$ & Pathogenic (chinchilla), $\mathrm{pYV}^{+}$ \\
\hline Y. enterocolitica IP 178 & $O: 2 a, 2 b, 3(5)$ & Pathogenic (hare), $\mathrm{pYV}^{+}$ \\
\hline Y. enterocolitica WE $245 / 92$ & $O: 3(4)$ & Pathogenic, $\mathrm{pYV}^{+}$ \\
\hline Y. enterocolitica ID & $0: 3(4)$ & Pathogenic, $\mathrm{pYV}^{+}$ \\
\hline Y. enterocolitica WA 289 & $\mathrm{O}: 8(1 \mathrm{~B})$ & Pathogenic, $\mathrm{pYV}^{+}$ \\
\hline Y. enterocolitica WA & $0: 8(1 B)$ & Pathogenic, $\mathrm{pYV}^{+}$ \\
\hline Y. enterocolitica WE 517/91 & $0: 9(2)$ & Pathogenic, $\mathrm{pYV}^{+}$ \\
\hline Y. enterocolitica PT & $0: 9(2)$ & Pathogenic, $\mathrm{pYV}^{+}$ \\
\hline Y. pseudotuberculosis WE 23/90 & I & Pathogenic, $\mathrm{pYV}^{+}$ \\
\hline Y. pseudotuberculosis WS 41/91 & I & Pathogenic, $\mathrm{pYV}^{+}$ \\
\hline Y. pseudotuberculosis WS 45/91 & II & Pathogenic, $\mathrm{pYV}^{+}$ \\
\hline Y. pseudotuberculosis WS 66/89 & III & Pathogenic, $\mathrm{pYV}^{+}$ \\
\hline Y. pestis KIM & & Avirulent, $\mathrm{pYV}^{-}$ \\
\hline S. minnesota $\operatorname{Re} 595$ & & Deep R-LPS ( $\operatorname{Re}$ chemotype) \\
\hline S. minnesota $\mathrm{Rd}_{1} \mathrm{p}+$ & & Deep R-LPS ( $\mathrm{Rd}_{1}$ chemotype) \\
\hline E. coli & $\mathrm{O} 111 \mathrm{~K} 58 \mathrm{H} 2$ & Enteropathogenic \\
\hline
\end{tabular}

Table 2. MICs of hydrophobic agents

\begin{tabular}{|c|c|c|c|c|c|c|c|c|}
\hline \multirow{2}{*}{$\begin{array}{l}\text { Hydrophobic } \\
\text { agent (partition } \\
\text { coefficient) }\end{array}$} & \multicolumn{8}{|c|}{ MIC $\left(\mu \mathrm{g} \mathrm{ml}^{-1}\right)$ for $†$} \\
\hline & $\begin{array}{l}\text { Y. enterocolitica } \\
\text { WA } 2890: 8\end{array}$ & $\begin{array}{c}\text { Y. pseudotuberculosis } \\
\text { WE } 23 / 90\end{array}$ & $\begin{array}{c}Y \cdot p \text { seudotuberculosis } \\
\text { WS } 41 / 91\end{array}$ & $\begin{array}{c}\text { Y. pseudotuberculosis } \\
\text { WS 45/91 }\end{array}$ & $\begin{array}{c}Y . p \text { seudotuberculosis } \\
\text { WS } 66 / 89\end{array}$ & $\begin{array}{l}\text { Y.pestis } \\
\text { KIM }\end{array}$ & $\begin{array}{l}\text { S. minnesota } \\
\operatorname{Rd}_{1} \mathrm{p}+\end{array}$ & $\begin{array}{l}\text { S. minnesota } \\
\text { Re595 }\end{array}$ \\
\hline Crystal violet (14·4) & 256 & 64 & 16 & 8 & 16 & 32 & $<2$ & $<2$ \\
\hline Malachite green $(4 \cdot 2)$ & 32 & 8 & 16 & 8 & 8 & 8 & $<2$ & $<2$ \\
\hline Novobiocin $(>20)$ & 256 & 16 & 32 & 32 & 64 & 32 & $<2$ & 8 \\
\hline Nafcillin $(0.31)$ & 256 & 32 & 32 & 32 & 64 & 64 & 64 & 64 \\
\hline Brilliant green & 2 & ND & 2 & 2 & 8 & ND & $<2$ & $<2$ \\
\hline
\end{tabular}

*Partition coefficient in 1-octanol/0.05 M sodium phosphate (pH 7.0) at $24^{\circ} \mathrm{C}$ (taken from Nikaido, 1976).

† ND, not done.

stored at $4{ }^{\circ} \mathrm{C}$ for several hours before analysis. Measurements were performed with a Bruker IFS 55 apparatus as previously described (Brandenburg \& Seydel, 1990). Briefly, LPS dispersions (water content $>90 \%$ ) were analysed in $\mathrm{CaF}_{2}$ cuvettes with $12.5 \mu \mathrm{m}$ thick Teflon spacers. For each measurement 50 interferograms were accumulated, Fouriertransformed and converted to absorbance spectra. The measurements were performed in continuous heating scans $\left(2{ }^{\circ} \mathrm{C} \mathrm{min} \mathrm{m}^{-1}\right)$ from 10 to $60^{\circ} \mathrm{C}$. The peak position of the symmetric stretching vibration of the methylene groups, $v_{\mathrm{s}}\left(\mathrm{CH}_{2}\right)$, around $2850 \mathrm{~cm}^{-1}$ was taken as a measure of the state of order (fluidity) of the acyl chains (Casal \& Mantsch, 1984). (ii) Fluorescence polarization (P). LPS dispersions were prepared as described for the FTIR experiments and labelled by incubation for a few minutes in $1 \mathrm{mM}$ diphenylhexatriene. Measurements were performed with a Kontron SFM 25 fluorescence spectrometer. The dye was excited by polarized light at $360 \mathrm{~nm}$ and the emission intensities (I) at $420 \mathrm{~nm}$ were measured simultaneously parallel $(\|)$ and perpendicular $(\perp)$ to the polarization plane of the incident beam. For each measurement, the emission was corrected by the grating factor, $G=I_{\perp} / I_{\|}$. Fluorescence polarization, $P=$ $\left(I_{\|}-I_{\perp}\right) /\left(I_{\|}+I_{\perp}\right)$, was determined as a function of temperature from 10 to $60^{\circ} \mathrm{C}$ at a heating rate of $3{ }^{\circ} \mathrm{C} \mathrm{min}^{-1}$. 


\section{RESULTS}

\section{Sensitivity of Yersinia spp. to hydrophobic antibiotics and dyes}

The Y. enterocolitica and Y. pseudotuberculosis strains and $Y$. pestis KIM (Table 1) were grown in tryptic soy broth at $26^{\circ} \mathrm{C}$ for $18 \mathrm{~h}$ and $20 \mu \mathrm{l}$ aliquots of these fresh inocula were seeded on MacConkey, SalmonellaShigella and brilliant green agars (Difco) and incubated at 26 or $37^{\circ} \mathrm{C}$ for $24 \mathrm{~h}$. All Y. enterocolitica strains grew on both MacConkey and SalmonellaShigella agars, regardless of the temperature. By contrast, Y. pestis KIM was completely inhibited in both media at 26 and $37^{\circ} \mathrm{C}$. All Y. pseudotuberculosis grew on MacConkey agar at 26 and $37^{\circ} \mathrm{C}$, but they produced only pin-point colonies on Salmonella-Shigella agar at $26^{\circ} \mathrm{C}$ and there was no growth at $37^{\circ} \mathrm{C}$. All yersiniae were completely inhibited on brilliant green agar at both 26 and $37^{\circ} \mathrm{C}$.

The MICs of hydrophobic dyes and antibiotics to representative $Y$. enterocolitica, $Y$. pseudotuberculosis and $Y$. pestis strains are shown in Table 2. With the exception of brilliant green, Y. pseudotuberculosis and $Y$. pestis were more sensitive to those agents than $Y$. enterocolitica, but they were not as sensitive as the deep rough (R) S. minnesota.

\section{NPN uptake}

Representative results obtained with cells grown at $37^{\circ} \mathrm{C}$ are presented in Fig. 1. In the absence of membrane metabolic inhibitors, pathogenic (WA 289 O:8) and non-pathogenic (MA 279 O:5) Y. enterocolitica took up only a limited amount of the probe (up to 25 RFU), whereas a marked uptake was observed for Y. pseudotuberculosis WE 23/90 and Y. pestis KIM in particular. These relative differences did not change when the remaining $Y$. enterocolitica and $Y$. pseudotuberculosis strains were tested. Moreover, Y. kristensenii WA 396, Y. intermedia WA 9/91, Y. frederiksenii WA 99/92, Y. bercovieri WS 68/91, Y. mollaretii WA 19/88 and Y. rohdei WA 9/90 yielded results identical to those of $Y$. enterocolitica (not shown). As expected, E. coli showed NPN uptake similar to that of Y.enterocolitica strains, whereas S. minnesota Re595 took up more probe (not shown). Although the NPN uptake of Y. pseudotuberculosis and $Y$. pestis was slightly reduced, similar observations were made with all strains in Table 1 grown at $26^{\circ} \mathrm{C}$.

The action of efflux systems was tested using nonpathogenic $Y$. enterocolitica and pathogenic yersiniae strains shown in Table 1 (grown at $37^{\circ} \mathrm{C}$ ) with $\mathrm{KCN}$ and sodium arsenate as inhibitors (Berger, 1973), used simultaneously at $1-25 \mathrm{mM}$ concentrations. Results with $10 \mathrm{mM} \mathrm{KCN}$ plus $10 \mathrm{mM}$ sodium arsenate and representative strains are presented in Fig. 1(b). It can be seen that inhibitors had no detectable effect on NPN uptake by $Y$. pseudotuberculosis and Y. pestis and that they caused NPN to be taken up by non-pathogenic and pathogenic Y. enterocolitica. These effects were maximal at concentrations as low as $1 \mathrm{mM}$ for pathogenic Y.enterocolitica and $10 \mathrm{mM}$ for non-pathogenic strains, with identical kinetics of uptake and final fluorescence values for higher concentrations. Fig. 1 also shows that the rate of NPN uptake was highest for $Y$. pestis and $Y$. pseudotuberculosis (185 and 115 RFU, respectively, in (a)

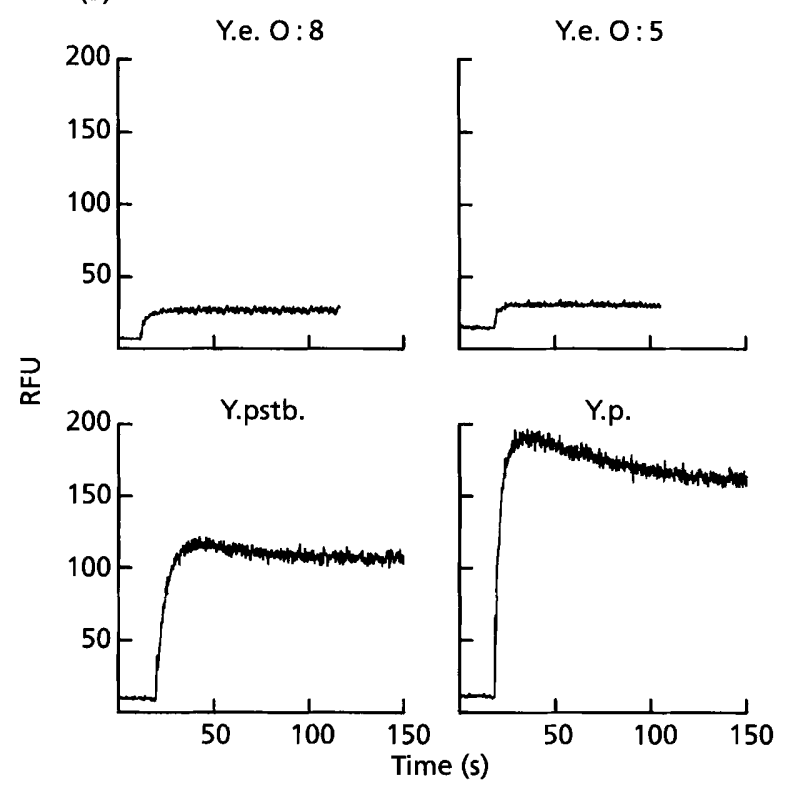

(b)

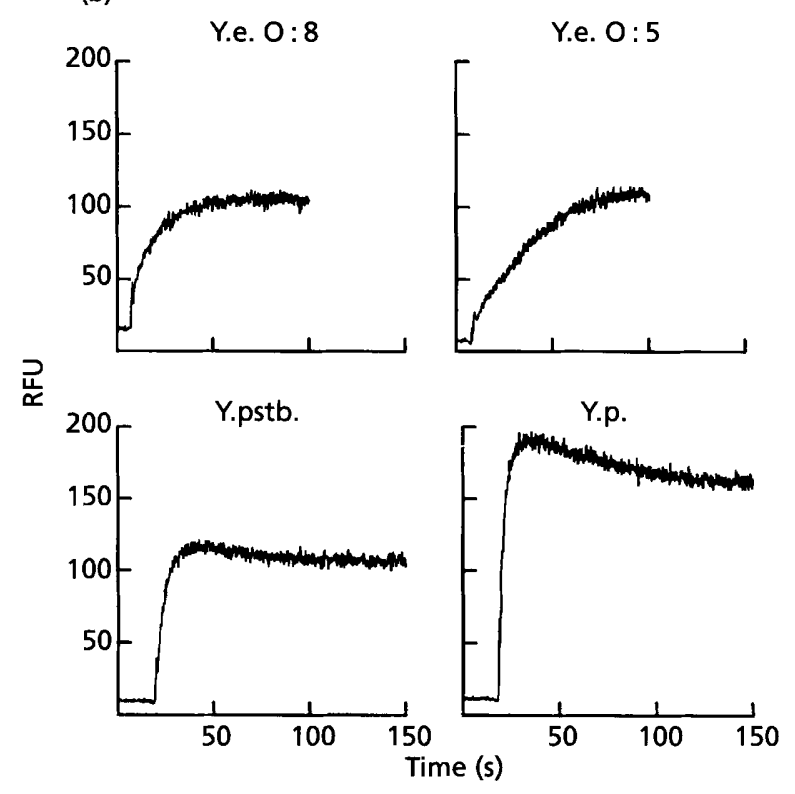

Fig. 1. OM permeability to NPN in Y. enterocolitica MA 279 0:5 (Y.e. 0:5), Y. enterocolitica WA 289 0:8 (Y.e. 0:8), Y. pseudotuberculosis WE 23/90 (Y.pstb.) and Y. pestis KIM (Y.p.). Cells were grown at $37^{\circ} \mathrm{C}$ and fluorescence monitored with (a) or without (b) membrane metabolic inhibitors. 
(a)

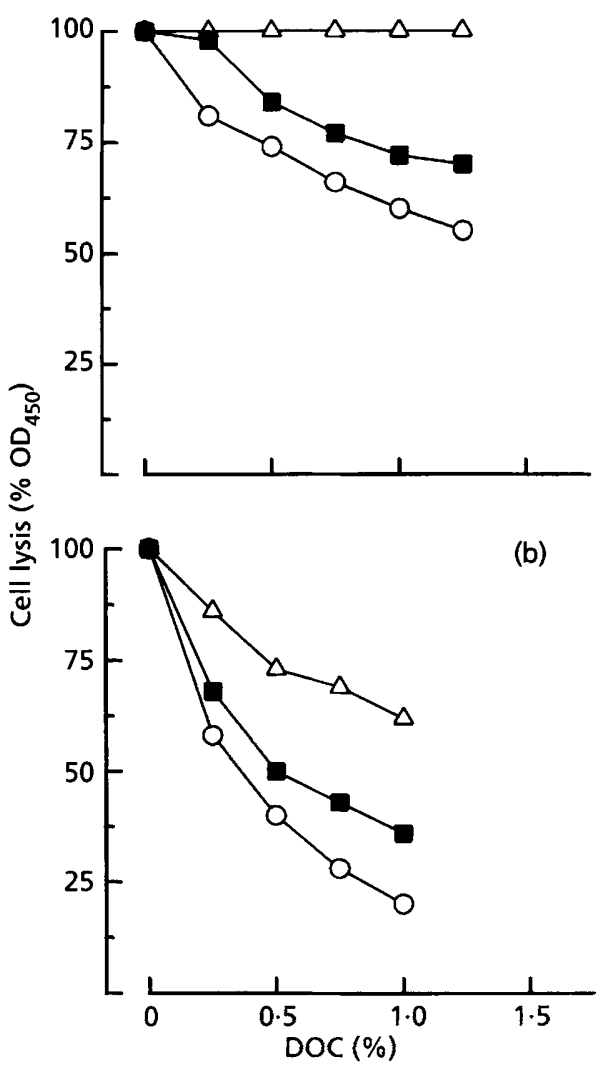

Fig. 2. Cell lysis induced by DOC. Y. enterocolitica WA $2890: 8$ $(\triangle), Y$. pseudotuberculosis WE $23 / 90(\square)$ and $Y$. pestis KIM $(O)$ grown at $37^{\circ} \mathrm{C}$ were incubated with DOC without (a) or with (b) membrane metabolic inhibitors and the decrease in $O D_{450}$ was recorded after $1 \mathrm{~h}$ incubation at $37^{\circ} \mathrm{C}$. Results are the mean of two independent experiments (coefficient of variation less than $5 \%$ for all groups of data).

$5 \mathrm{~s})$, intermediate for pathogenic Y. enterocolitica (110 RFU in $45 \mathrm{~s}$ ) and lowest for non-pathogenic Y. enterocolitica (110 RFU in $90 \mathrm{~s}$ ). Y. pseudotuberculosis WE 23/90 and Y. enterocolitica WA $289 \mathrm{O}: 8 \mathrm{pYV}^{+}$and pYV ${ }^{-}$pairs yielded similar results both with and without inhibitors and at either 26 or $37^{\circ} \mathrm{C}$ (not shown).

\section{Sensitivity of Yersinia spp. to DOC}

With cells growing exponentially at $37^{\circ} \mathrm{C}$, DOCinduced lysis in the absence of metabolic inhibitors was greater for Y. pestis KIM than for Y. pseudotuberculosis WE 23/90 and no lysis was observed for Y. enterocolitica WA 289 O:8 (Fig. 2a). Membrane metabolic inhibitors (at concentrations having maximal effect in the NPN experiments) rendered these three yersiniae susceptible to DOC-induced lysis, but the relative differences in susceptibility were maintained (Fig. 2b). Similar results were obtained with the remaining $Y$. pseudotuberculosis strains (not shown) and none of them was as sensitive to DOC as Y. pestis KIM. Although lysis was less marked, similar observations

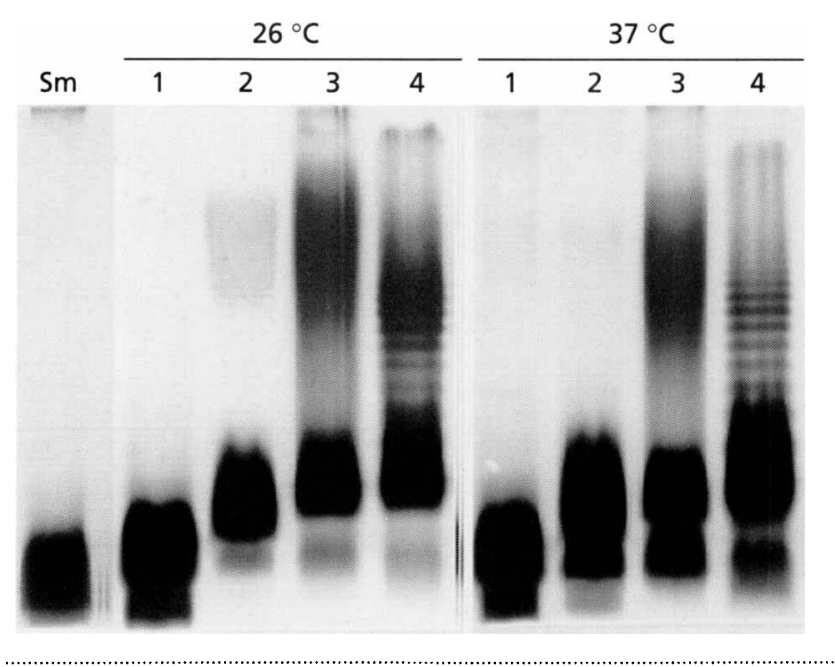

Fig. 3. SDS-PAGE followed by silver staining of LPS preparations $(10 \mu \mathrm{g})$ from cells grown at the indicated temperature. Lanes: 1 , Y. pestis KIM; 2, Y. pseudotuberculosis WE 23/90; 3, Y. enterocolitica WE 245/92 0:3; 4, Y. enterocolitica WA 289 0:8. The deep R-LPS from S. minnesota Re595 (lane Sm) is included as a reference.

were made with cells grown at $26^{\circ} \mathrm{C}$ (not shown). Interestingly, stationary phase cells of Y. pseudotuberculosis and $Y$. pestis were more sensitive to DOC than exponential phase cells; this effect was not observed with Y. enterocolitica.

\section{LPS characterization}

Fig. 3 shows SDS-PAGE profiles of the LPSs from $Y$. enterocolitica WA 289 O:8 and WE 245/92 O:3, Y. pseudotuberculosis WE 23/90 and Y. pestis KIM cells grown at 26 and $37^{\circ} \mathrm{C}$. The Y. enterocolitica LPSs showed both short and long polysaccharide molecules typical of smooth (S)-LPS and the absence (WE 245/92 O:3 LPS) or presence (WA 289 O:8 LPS) of the ladderlike pattern that relates to the number of sugars in the $\mathrm{O}$ chain repetitive units (Hoffman et al., 1980; Tomshich et al., 1987). Y. enterocolitica PR O:1,6 (non-pathogenic) also had an S-LPS, but with no conspicuous ladder-like pattern (not shown). These analyses also showed enrichment of the low molecular mass components at $37^{\circ} \mathrm{C}$, with a conspicuous fast-migrating band (Fig. 3). Y. pseudotuberculosis WE 23/90 LPS only showed traces of long polysaccharide LPS when grown at $26^{\circ} \mathrm{C}$ and also showed enrichment of a fast-migrating component at $37^{\circ} \mathrm{C}$ (Fig. 3). Finally, Y. pestis KIM LPS was similar at 26 and $37^{\circ} \mathrm{C}$ and contained a component of mobility similar to that of the fast-migrating component of Y. pseudotuberculosis plus a second component migrating closer to the running front of the gel (Fig. 3). The Kdo contents of the LPSs at 26 and $37^{\circ} \mathrm{C}$ were, respectively, $2 \cdot 28$ and $2 \cdot 84 \%$ for Y. enterocolitica WA 289 O : $8,1.95$ and $2.80 \%$ for Y. enterocolitica WE $245 / 92$ O:3, 1.14 and $2.03 \%$ for Y. enterocolitica PR $\mathrm{O}: 1,6,2 \cdot 47$ and $5 \cdot 65 \%$ for Y. pseudotuberculosis WE 
(a)

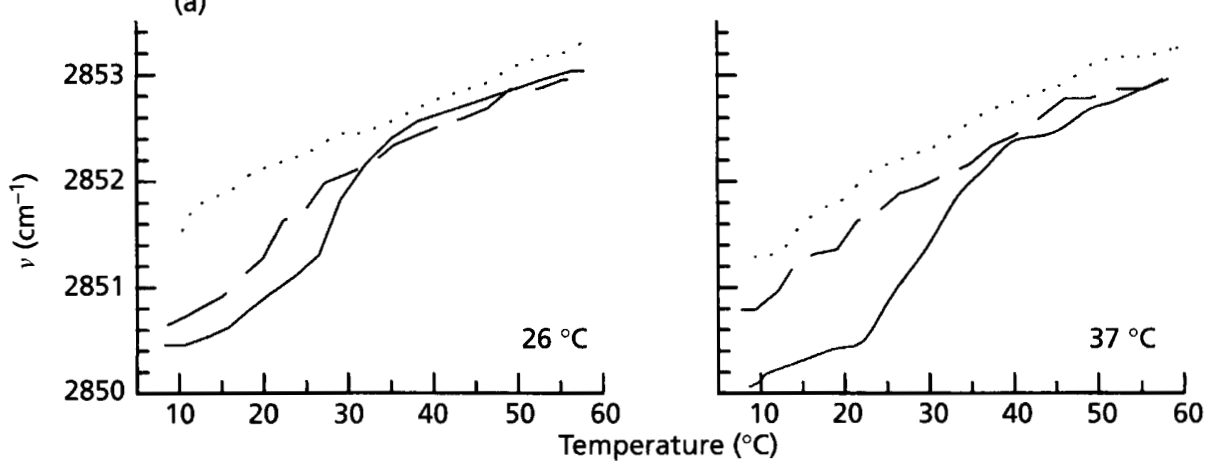

(b)

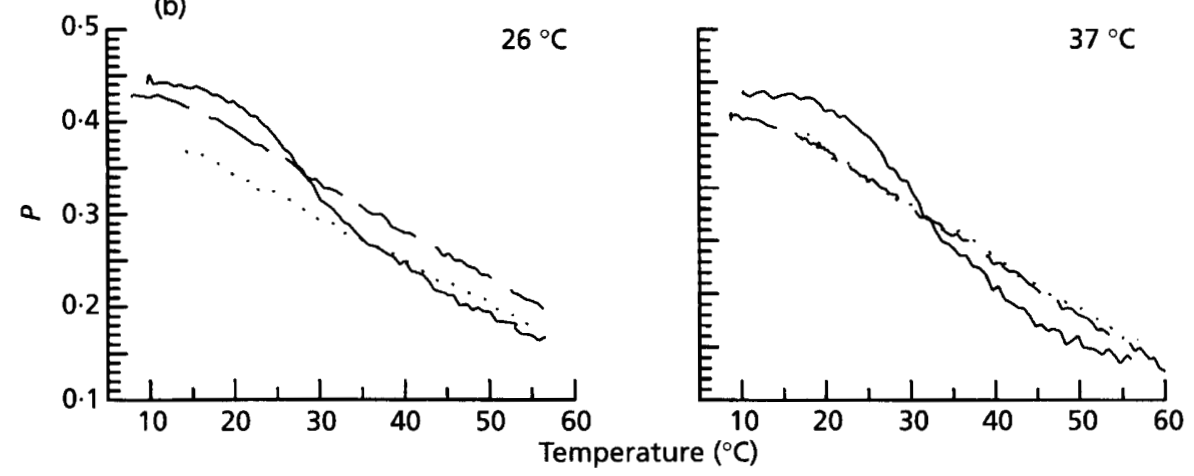

Fig. 4. Temperature dependence of (a) maximum of the peak position of the symmetric stretching vibration, $v_{s}\left(\mathrm{CH}_{2}\right)$, and (b) fluorescence polarization, $P$, in LPSs of $Y$. enterocolitica WA 289 0:8 (continuous lines), $Y$. pseudotuberculosis WE $23 / 90$ (dashed lines) and $Y$. pestis KIM (dotted lines) grown at 26 or $37^{\circ} \mathrm{C}$.

$23 / 90$, and 6.84 and $8.08 \%$ for $Y$. pestis KIM. No phospholipid was detected in these preparations and protein contents were below $1 \cdot 0 \%$.

For many biological process, e.g. interactions at cell membrane level, acyl-chain fluidity is known to play an important role. For this, we determined the $\beta \leftrightarrow \alpha$ phase transition of the hydrocarbon chains of LPS preparations from cells grown at 26 and $37^{\circ} \mathrm{C}$. The temperature-dependent shift in the maximum of the peak position of the symmetric stretching vibration, $v_{\mathrm{s}}\left(\mathrm{CH}_{2}\right)$, of LPS aggregates is presented in Fig. 4(a). For the Y.enterocolitica WA 289 O:8 LPS and regardless of the growth temperature, the peak positions were in the 2850.0 to $2850.5 \mathrm{~cm}^{-1}$ range at temperatures below $15^{\circ} \mathrm{C}$ and over $2852.0 \mathrm{~cm}^{-1}$ above $35^{\circ} \mathrm{C}$. For this LPS, a clear $\beta \leftrightarrow \alpha$ phase transition in the $19-35^{\circ} \mathrm{C}$ interval was observed (Fig. 4a) with a $T_{\mathrm{c}}$ of $29^{\circ} \mathrm{C}$. The Y. enterocolitica WE 245/92 O:3 and PR O:1,6 LPSs yielded similar results (not shown) with $T_{\mathrm{c}}$ values from 28 to $31^{\circ} \mathrm{C}$. The results obtained with the LPS of Y. pseudotuberculosis WE $23 / 90$ grown at $26^{\circ} \mathrm{C}$ were close to those of the Y. enterocolitica LPSs, but no clear phase transition was observed (Fig. 4a). With the LPS of Y. pseudotuberculosis WE $23 / 90$ grown at $37^{\circ} \mathrm{C}$, the peak positions were higher over the whole temperature range, with no phase transition (Fig. 4a). The results obtained with the Y. pestis KIM LPS were similar for the two growth temperatures, with peak positions constantly higher than those obtained with the LPSs of the other Yersinia spp. and no phase transition (Fig. 4a).

Analysis of $\beta \leftrightarrow \alpha$ phase transitions was also carried out by measuring the change in fluorescence polarization, $P$, of diphenylhexatriene-labelled LPS aggregates as a function of temperature with results in good agreement with those obtained by FTIR. This second method showed clear phase transitions in LPS aggregates of $Y$. enterocolitica WA 289 O:8 grown at either 26 or $37^{\circ} \mathrm{C}$ with only a $2^{\circ} \mathrm{C}$ difference with respect to the $T_{\mathrm{c}}$ value obtained by FTIR (Fig. 4b). The LPSs of Y. enterocolitica WE 245/92 O:3 and PR O:1,6 yielded similar results (not shown) to those of the WA 289 O: 8 LPS, but no phase transitions were observed for the LPSs of $Y$. pseudotuberculosis WE 23/90 or Y. pestis KIM, irrespective of the growth temperature (Fig. 4b). For cells grown at $26^{\circ} \mathrm{C}$, and in agreement with the FTIR results, the $P$ values of the $Y$. psendotuberculosis WE 23/90 LPS were closer to those of the Y. enterocolitica LPSs at temperatures below the $T_{\mathrm{c}}$ of the latter and those of the $Y$. pestis KIM LPS indicated a fluid state over the whole temperature range (Fig. $4 \mathrm{~b}$ ). With cells grown at $37^{\circ} \mathrm{C}$, the fluidity values were similar for the LPSs of $Y$. pseudotuberculosis WE 23/90 and Y. pestis KIM. 


\section{DISCUSSION}

This study provides coincident indirect (growth differences on selective media and MICs) and direct (NPN uptake and DOC-induced cell lysis) evidence showing that Yersinia spp. vary in their ability to repel hydrophobic agents and surfactants. Moreover, agreement was found between the different observations with regard to variations associated with species or growth temperature. Accordingly, Y. pestis KIM was inhibited on selective media on which the remaining Yersinia spp. grew and it also showed the highest NPN accumulation and DOC sensitivity. Also, Y. pseudotuberculosis, which grew on Salmonella-Shigella agar at $26^{\circ} \mathrm{C}$ but not at $37^{\circ} \mathrm{C}$, showed intermediate NPN accumulation and DOC sensitivity, both of which were enhanced at $37^{\circ} \mathrm{C}$. All these data are in agreement with previous observations on the variable growth responses of $Y$. pestis and Y. pseudotuberculosis on MacConkey agar and, depending on the temperature, also on SalmonellaShigella agar (Bercovier \& Mollaret, 1984).

It has been shown that some Gram-negative bacteria are endowed with efflux systems that pump out hydrophobic permeants, thereby complementing the barrier function of the OM to different extents (Hancock, 1997; Nikaido, 1994, 1996). Since efflux systems use energy, increased accumulation of a given compound in the presence of membrane metabolic inhibitors is usually taken as indirect evidence for such a system (Hancock, 1997; Nikaido, 1994, 1996). In this work, KCN plus sodium arsenate were used, rather than uncouplers such as carbonyl cyanide $m$-chlorophenylhydrazone (CCCP), because we have observed that this agent causes artefacts in bacteria having OMs permeable to hydrophobic agents (A. Sola, J. A. Bengoechea \& I. Moriyón, unpublished) and artefacts in membrane studies have also been observed by others (Hancock \& Braun, 1976; Helgerson \& Cramer, 1977). Since the net entry of hydrophobic compounds into cells of Yersinia spp. was increased by inhibitors not having side effects, the results strongly suggest that efflux systems are more efficient in $Y$. enterocolitica and in non-pathogenic strains in particular, than in Y. pseudotuberculosis or Y. pestis. With Y.pseudotuberculosis and Y.pestis, inhibitors had no apparent effect in the NPN uptake test, but the very high rate of spontaneous uptake could mask possible efflux systems. In fact, the presence of efflux systems in Y. pseudotuberculosis and Y. pestis is suggested by the increase of DOC-induced cell lysis observed with metabolic inhibitors. Alternatively, this apparent discrepancy could reflect the specificity of the efflux systems, since DOC is chemically closer to permeants present in the host. However, differences in efflux systems cannot account for the observations by themselves. Even at optimal inhibitor concentrations, the net entry of both NPN and DOC decreased in the order $Y$. pestis-Y. pseudotuberculosis-pathogenic Y. enterocolitica-non-pathogenic $Y$. enterocolitica, suggesting that OM structural differences were also affecting the rate of partition of the permeants tested. Indeed, this is in agreement with the idea that efficient efflux systems call for efficient OM barriers.

The increase in $\mathrm{OM}$ permeability to hydrophobic compounds was paralleled both by a reduction in the LPS polysaccharide moiety and by an increase in acylchain fluidity. LPS molecules are located only in the outer leaflet of the OM and, at least in E. coli and Salmonella $\mathrm{S}$ cells, they are tightly bridged by divalent cations, thereby excluding phospholipids from the outer leaflet (Hancock, 1984; Nikaido \& Vaara, 1985). S. typhimurium $\mathrm{Rd}$ and $\mathrm{Re}$ (deep R) mutants and their equivalent $E$. coli D21f1 and D21f2 mutants, which have no or reduced heptose and phosphate in the LPS core, are hypersensitive to hydrophobic antibiotics and dyes. Since a role of the LPS O-specific polysaccharide is ruled out by the similar OM permeability of $S$ cells and $\mathrm{Ra}, \mathrm{Rb}$ and Rc mutants, the higher permeability of Rd and Re mutants can be explained by the presence of phospholipid molecules in the OM outer leaflet through which hydrophobic permeants can penetrate (Nikaido \& Vaara, 1985). In addition, penetration can occur through LPS-covered areas where the overall OM structure becomes altered as a consequence of the absence of core components necessary for effective bridging (Nikaido \& Vaara, 1985). Furthermore, it has been shown in synchrotron X-ray small angle diffraction studies that, under conditions under which LPS samples independent of the length of the sugar chain adopt lamellar structures (low water content and high $\mathrm{Mg}^{2+}$ concentration), the values for the lamellar repeat $d_{1}$ are $6.0-6.5 \mathrm{~nm}$ for $R e$ and Rd LPS, but increase considerably to more than $8.0 \mathrm{~nm}$ for Rc LPS (Seydel et al., 1993). From this, the hydration shells of Rc LPS and those LPSs with longer sugar chains can be assumed to be much larger than for the Rd and Re LPSs. Thus, in addition to the changed LPS-LPS interactions, the reduced hydration of Re or Rd LPS might explain the increased susceptibility (permeability) to hydrophobic drugs of the corresponding mutants. Phospholipid exposure at the OM surface has been clearly shown in $S$. typhimurium deep $\mathrm{R}$ mutants and, accordingly, direct permeation through OM phospholipid patches is the likely explanation for their increased OM permeability (Nikaido \& Vaara, 1985). However, this is not always the case because Brucella abortus (which carries an S-type LPS) readily takes up hydrophobic agents (Martínez de Tejada \& Moriyón, 1993; Freer et al., 1996) and there is evidence that this uptake can be explained on the sole basis of the properties of the LPS (Freer et al., 1996; Martínez de Tejada \& Moriyón, 1993).

The reduced polysaccharide content of Y. pseudotuberculosis LPSs could suggest a situation similar to that of the deep R S. minnesota and E. coli mutants. However, although they showed a growth temperaturedependent increase in the roughness of the LPS, the SDSPAGE analyses showed no component of mobility similar to the S. minnesota Re LPS in the LPS of Y. pseudotuberculosis WE 23/90 grown at either 26 or $37^{\circ} \mathrm{C}$. Those analyses also showed two overlapping 
components in the LPS of Y. pestis KIM, one of which could be equivalent to the Re595 S. minnesota LPS, while the other was similar to the rougher component detected in Y. pseudotuberculosis WE $23 / 90$ at $37^{\circ} \mathrm{C}$. Therefore, this strain cannot be considered as totally equivalent to the deep R $S$. minnesota strains. Although there are no chemical data for the LPSs of Y. pseudotuberculosis grown at $37^{\circ} \mathrm{C}$, the SDS-PAGE result is in agreement with the work of Samuelsson et al. (1973) who showed that the LPSs of Y. pseudotuberculosis serogroups I-V grown at $26^{\circ} \mathrm{C}$ carry an oligosaccharide stub attached to the outer core. Therefore, the evidence available suggests that the Y. pseudotuberculosis LPS is not truly $\mathrm{R}$ but similar to the lipo-oligosaccharides of Neisseria, Bordetella or Haemophilus spp. (Hitchcock et al., 1986). As discussed below, the acyl-chain fluidity analyses also showed important differences (absence or presence of transitions, respectively) between the LPSs of pathogenic yersiniae and deep R S. minnesota.

The acyl-chain fluidity analyses point to reduced LPSLPS interactions as a likely reason for the increased OM permeability to hydrophobic agents. Both FTIR and fluorescence polarization spectroscopy showed that (i) Y. pseudotuberculosis WE 23/90 and Y. pestis KIM LPSs formed aggregates with more fluid acyl chains than those of other Yersinia spp., (ii) Y. pestis KIM LPS aggregates had more fluid acyl chains than those of $Y$. pseudotuberculosis WE 23/90 and (iii) the acyl-chain fluidity of the LPSs from Y. pseudotuberculosis WE $23 / 90$ and Y. enterocolitica were closer when the cells were grown at $26^{\circ} \mathrm{C}$ than when grown at $37^{\circ} \mathrm{C}$. Obviously, these three sets of data match the NPN net uptake, DOC sensitivity, inhibition on selective media and effect of the growth temperature observed for live Yersinia spp cells. To the best of our knowledge, Y. enterocolitica, Y. pseudotuberculosis and Y. pestis are the first bacteria in which a positive correlation between LPS acyl-chain fluidity and OM permeability is established and, although experimental conditions or interaction with proteins can alter the fluidity in the intact $O M$, it seems rather unlikely that such values would not remain comparatively meaningful.

The variations in LPS acyl-chain fluidity (and the concomitant variations in LPS-LPS interactions) must have a structural explanation. Since electrostatic and/or hydrophilic interactions between polysaccharide moieties of LPSs can decrease the overall LPS fluidity (Brandenburg \& Seydel, 1984, 1990) and the LPS polysaccharide length decreased in the order $Y$. enterocolitica-Y.pseudotuberculosis-Y.pestis, this sort of effect could account in part for the increase in acylchain fluidity. Moreover, the same effects could explain the increase in acyl-chain fluidity observed for the LPS of Y. pseudotuberculosis grown at $37^{\circ} \mathrm{C}$. However, as phase transitions have been observed for R LPSs of enterobacteria under conditions similar to those used in this study (Brandenburg \& Seydel, 1984, 1990), their absence in the LPSs of Y. pseudotuberculosis and Y. pestis is intriguing and cannot be explained on the sole basis of a reduced interaction between poly or oligo- saccharide LPS moieties. The fluidity of the Y. pseudotuberculosis, Y. pestis and Y. enterocolitica LPS acyl chains were very close at temperatures above the $T_{\mathrm{c}}$ of the latter LPS. This shows that the absence of transitions in the LPSs of Y. pseudotuberculosis and Y. pestis reflects a constant liquid-crystalline state and an explanation for this is a lower intensity of the hydrophobic effect at the lipid A level. Thus, a lipid A structure different from that of better characterized enterobacteria can be predicted for Y. pseudotuberculosis and Y. pestis. In fact, Hartley et al. (1974) have reported an increased proportion of hydroxylated fatty acids with respect to other fatty acids in the LPS of Y. pestis and suggested that this should correspond to a lipid A with a structure more simple than that of other enteric bacteria.

In a previous study we have shown that the OMs of $Y$. enterocolitica pathogenic biogroups, but not those of non-pathogenic biogroups, become permeable to hydrophobic agents when grown with $\mathrm{Mg}^{2+}$ but no $\mathrm{Ca}^{2+}$ available (Bengoechea et al., 1996). As invasiveness is nil in non-pathogenic yersiniae, reduced in pathogenic $Y$. enterocolitica, significant in Y. pseudotuberculosis and high in Y. pestis (Brubaker, 1991; Mäki et al., 1983), OM permeability and efflux systems within the genus seem to correlate to habitat. Because of the presence of bile surfactants and enzymes, an OM impermeable to such agents is useful for bacteria colonizing the intestine or entering into the host tissues by this route and this correlates the $\mathrm{OM}$ impermeability of pathogenic $Y$. enterocolitica grown under standard conditions. In keeping with this and with our observations with $Y$. pseudotuberculosis, the lethal dose of Y. enterocolitica is over a thousand-fold lower than that of Y. pseudotuberculosis when they are administered orally (Pepe \& Miller, 1993; Rosqvist et al., 1988). However, as illustrated by the results obtained with both the environmental Yersinia spp. and the non-pathogenic biogroups of Y. enterocolitica, OMs are also impermeable in bacteria from non-enteric habitats in which surfactants or enzymes are not significant. Thus, the gradual increase in OM permeability of the pathogenic Y. enterocolitica-Y.pseudotuberculosis-Y. pestis series cannot be unequivocally explained as the loss of a property which becomes useless in a non-enteric environment. Although alternative explanations remain speculative, a permeable OM could be advantageous for bacteria living within host tissues and cells since exchange of hydrophobic nutrients and metabolites could be facilitated. Research with mutants showing altered OM permeability is in progress to test some of the above hypotheses.

\section{ACKNOWLEDGEMENTS}

We are grateful to H. Lüthje, C. Hamann and G. von Busse for technical assistance and to M. Alvarez-Marqués, E. J. Bottone, R. R. Brubaker, I. Dorronsoro, M. P. Doyle, G. Pratts and G. Wauters for providing some of the Yersinia strains. We also wish to express our gratitude to an anonymous reviewer for suggestions on the efflux experiments. Part of this work was financially supported by the Deutsche Forschungsgemein- 
schaft (SFB 470) and a PIUNA grant from the University of Navarra. Fellowship support for J.A.B. from the Eusko Jaurlaritza and Friends of the University of Navarra is also gratefully acknowledged.

\section{REFERENCES}

Bengoechea, J. A., Díaz, R. \& Moriyón, I. (1996). Outer membrane differences between pathogenic and environmental Yersinia enterocolitica biogroups probed with hydrophobic permeants and polycationic peptides. Infect Immun 64, 4891-4899.

Bercovier, H. \& Mollaret, H. H. (1984). Genus XIV. Yersinia Van Loghem 1944, 15 ${ }^{\mathrm{A}}$. In, Bergey's Manual of Systematic Bacteriology, vol. 1, pp. 498-506. Edited by N. R. Krieg \& J. G. Holt. London: Williams \& Wilkins.

Berger, E. A. (1973). Different mechanisms of energy coupling for the active transport of proline and glutamine in Escherichia coli. Proc Natl Acad Sci USA 70, 1514-1518.

Brandenburg, K. \& Seydel, U. (1984). Physical aspects of structure and function of membranes made from lipopolysaccharides and free lipid A. Biochim Biophys Acta 775, 225-238.

Brandenburg, K. \& Seydel, U. (1990). Investigation into the fluidity of lipopolysaccharide and free lipid A membrane systems by Fourier-transform infrared spectroscopy and differential scanning calorimetry. Eur J Biochem 191, 229-236.

Brubaker, R. R. (1991). Factors promoting acute and chronic diseases caused by yersiniae. Clin Microbiol Rev 4, 309-324.

Casal, H. L. \& Mantsch, H. H. (1984). Polymorphic phase behaviour of phospholipid membranes studied by infrared spectroscopy. Biochim Biophys Acta 779, 381-401.

Cornelis, G. R. \& Wolf-Watz, H. (1997). The Yersinia Yop virulon: a bacterial system for subverting eukaryotic cells. Mol Microbiol 23, 861-867.

Díaz-Aparicio, E., Aragón, V., Marín, C., Alonso, B., Font, M., Moreno, E., Pérez-Ortiz, S., Blasco, J. M., Dlaz, R. \& Moriyón, I. (1993). Comparative analysis of Brucella serotype $A$ and $M$ and Yersinia enterocolitica O:9 polysaccharides for serological diagnosis of brucellosis in cattle, sheep and goats. J Clin Microbiol 31, 3136-3141.

Freer, E., Moreno, E., Moriyón, I., Pizarro-Cerdá, J., Weintraub, A. \& Gorvel, J.P. (1996). Brucella/Salmonella-lipopolysaccharide chimeras are less permeable to hydrophobic probes and more sensitive to cationic peptides and EDTA than their native Brucella spp. counterparts. J Bacteriol 178, 5867-5876.

Hancock, R. E. W. (1984). Alterations in outer membrane permeability. Annu Rev Microbiol 38, 237-264.

Hancock, R. E. W. (1997). The bacterial outer membrane as a drug barrier. Trends Microbiol 5, 37-42.

Hancock, R. E. W. \& Braun, V. (1976). Nature of the energy requirement for the irreversible adsorption of bacteriophages T1 and phi80 to Escherichia coli. J Bacteriol 125, 409-415.

Hartley, J. L., Adams, G. A. \& Tornabene, T. G. (1974). Chemical and physical properties of lipopolysaccharide of Yersinia pestis. J Bacteriol 118, 848-854.

Helgerson, S. L. \& Cramer, W. A. (1977). Changes in Escherichia coli cell envelope structure and the sites of fluorescence probe binding caused by carbonyl cyanide $p$-trifluoromethoxyphenylhydrazone. Biochemistry 16, 4109-4116.

Hitchcock, P., Leive, L., Mäkela, P. H., Rietschel, E. T., Strittmatter, W. \& Morrison, D. C. (1986). Lipopolysaccharide nomenclature. Past, present and future. J Bacteriol 166, 699-705.
Hoffman, J., Lindberg, B. \& Brubaker, R. R. (1980). Structural studies on the $\mathrm{O}$-specific side-chains of the lipopolysaccharide from Yersinia enterocolitica Ye 128. Carbohydr Res 78, 212-214.

Loh, B., Grant, C. \& Hancock, R. E. W. (1984). Use of the fluorescent probe $1-N$-phenylnaphthylamine to study the interactions of aminoglycoside antibiotics with the outer membrane of Pseudomonas aeruginosa. Antimicrob Agents Chemother 26, 546-551.

Mäki, M., Vesikari, T., Rantala, I., Sundquist, C. \& Grönroos, P. (1983). Pathogenicity of 42-44 Mdal plasmid positive and negative Yersinia pseudotuberculosis I and Yersinia enterocolitica O:8 and $O: 9$ studied in the guinea pig eye model (Serény test). Acta Pathol Microbiol Immunol Scand 91, 241-244.

Markwell, M. A. K., Haas, S. M., Bieber, L. L. \& Tolbert, N. E. (1978). A modification of the Lowry procedure to simplify protein determination in membrane lipoprotein samples. Anal Biochem 87, 206-210.

Martínez de Tejada, G. \& Moriyón, I. (1993). The outer membranes of Brucella spp. are not barriers to hydrophobic permeants. J Bacteriol 175, 5273-5275.

Nikaido, H. (1976). Outer membrane of Salmonella typhimurium. Transmembrane diffusion of some hydrophobic substances. Biochim Biophys Acta 433, 118-132.

Nikaido, H. (1994). Prevention of drug access to bacterial targets : permeability barriers and active efflux. Science 264, 382-388.

Nikaido, H. (1996). Multidrug efflux pumps of Gram-negative bacteria. J Bacteriol 178, 5853-5859.

Nikaido, H. \& Vaara, M. (1985). Molecular basis of bacterial outer membrane permeability. Microbiol Rev 49, 1-32.

Pepe, J. C. \& Miller, V. L. (1993). Yersinia enterocolitica invasin: a primary role in the initiation of infection. Proc Natl Acad Sci USA 90, 6473-6477.

Riley, G. \& Toma, S. (1989). Detection of pathogenic Yersinia enterocolitica by using Congo red-magnesium oxalate agar medium. J Clin Microbiol 27, 213-214.

Rosqvist, R., Skurnik, M. \& Wolf-Waltz, H. (1988). Increased virulence of Yersinia pseudotuberculosis by two independent mutations. Nature 334, 522-525.

Samuelsson, K., Lindberg, B. \& Brubaker, R. R. (1973). Structure of O-specific chains of lipopolysaccharides from Yersinia pseudotuberculosis. J Bacteriol 117, 1010-1016.

Seydel, U., Koch, M. H. J. \& Brandenburg, K. (1993). Structural polymorphism of rough mutant lipopolysaccharides $\mathrm{Rd}$ to $\mathrm{Ra}$ from Salmonella minnesota. J Struct Biol 110, 232-243.

Sprott, G. D., Koval, S. F. \& Schnaitman, C. A. (1994). Cell fractionation. In Methods for General and Molecular Bacteriology, pp. 72-103. Edited by P. Gerhardt, R. G. E. Murray, W. A. Boot \& N. R. Krieg. Washington, DC: American Society for Microbiology.

Straley, S. C. \& Harmon, P. A. (1984a). Growth in mouse peritoneal macrophages of Yersinia pestis lacking established virulence determinants. Infect Immun 45, 649-654.

Straley, S. C. \& Harmon, P. A. (1984b). Yersinia pestis grows within phagolysosomes in mouse peritoneal macrophages. Infect Immun 45, 655-659.

Straley, S. C. \& Perry, R. (1995). Environmental modulation of gene expression and pathogenesis in Yersinia. Trends Microbiol 3, 310-317.

Thiele, O. W. \& Schwinn, G. (1973). The free lipids of Brucella melitensis and Bordetella pertussis. Eur J Biochem 34, 333-344. 
Tomshich, S. V., Gorshkova, R. P. \& Ovodov, Y. S. (1987). Structure studies on lipopolysaccharide from $Y$. enterocolitica serovar O:8. Khim Prir Soedin 657-664.

Träuble, H. \& Overath, P. (1973). The structure of Escherichia coli membranes studied by fluorescence measurements of lipid phase transitions. Biochim Biophys Acta 307, 491-512.
Tsai, C.-M. \& Frasch, C. E. (1982). A sensitive silver stain for detecting lipopolysaccharide in polyacrylamide gels. Anal Biochem 119, 115-119.

Received 3 July 1997; revised 8 December 1997; accepted 2 March 1998. 